\title{
Normalization of Insulin resistance, Glucose intolerance and Lipid profile by Swietenia mahagoni leaf extract in fructose induced diabetic rats
}

\author{
Naveen Y Puttaswamy ${ }^{1}$, Asna Urooj ${ }^{2}$ \\ ${ }^{1}$ Department of Biochemistry, Pooja Bhagavath Mahajana PG center, Metagalli, Mysore, Karnataka, India-570016, \\ India, ${ }^{2}$ Department of Food Science and Nutrition, University of Mysore, Mysore-570006, India
}

Swietenia mahagoni (L.) Jacq. is a medicinal plant used in traditional medicinal practices for the treatment of various human ailments. The plant has been shown to possess potent pharmacological value in our previous studies. The present study is an extension of anti-diabetic study in type 2 diabetic model.

Methods

Present study evaluates the anti-diabetic potential of Swietenia mahagoni (L.) Jacq. aqueous extract (MAE) in fructose induced diabetic rats with insulin resistance. Insulin resistance was induced in Wistar rats by feeding fructose $(20 \%$ W/V) for 8 week. After the confirmation of insulin resistance induction, the rats were treated with MAE for 45 days and the effect of treatment on the diabetes pathology was continuously assessed. MAE was analyzed using HPLC and LCMS for identification of constituent bio-actives present.

Results

MAE treatment improved glucose levels by $64.10 \%$, reduced the insulin levels by $27.56 \%$ insulin resistance by $52.2 \%$, improved oral glucose tolerance, improved pancreatic beta cell health by $29.5 \%$, decreased glycated hemoglobin content by $23.28 \%$, reduced lipid peroxides, improved glycogen content by $21.8 \%$, overall improvement in antioxidant status and improved lipid profile in treated group when compared to diabetic control group. The HPLC analysis indicated the presence of gallic acid, scopoletin, coumarin, epicatechin and ellagic acid and their presence was confirmed by LCMS analysis.

Conclusion

Results supported the anti-diabetic potential of MAE in type 2 diabetic model, where it acts on multi-targets and ameliorate the overall diabetic condition rather only targeting to control hyperglycemia. Thus the study supports the scope of Swietenia mahagoni (L.) Jacq as a potential candidate which can be studied as adjuvant in Type 2 diabetic subjects. 